\title{
Enfoque AICLE y enseñanza del español en la educación superior británica: perspectivas del profesorado de ELE
}

\author{
Content and Language Integrated Learning (CLIL) and Spanish \\ Language Teaching in British Higher Education: perspectives \\ from language lecturers
}

\author{
CARMEN FLORIANO RAMOS \\ UNIVERSIDAD CAMILO JosÉ CELA \\ carmen.florianoramos@gmail.com \\ https://orcid.org/0000-0002-6769-9659 \\ MARA FueRTES GutIÉRREZ \\ THE OPEN UNIVERSITY \\ mara.fuertes-gutierrez@open.ac.uk \\ https://orcid.org/0000-0002-9890-5945
}

\begin{abstract}
Resumen
En la educación superior en Reino Unido, en los planes de estudio donde se enseña español, se distingue entre asignaturas denominadas de lengua - donde se aprende el idioma- y las denominadas "de contenido" -que tratan la historia, la política o la literatura en los países donde se habla español y se imparten en inglés-. Este artículo se apoya en el análisis temático de entrevistas a profesores universitarios de ELE en Reino Unido para explorar si el empleo del enfoque AICLE (Aprendizaje integrado de Contenido y Lenguas Extranjeras) puede facilitar la colaboración entre docentes de ambos tipos de asignaturas.

Palabras clave: Enseñanza del español; Enfoque AICLE (Aprendizaje integrado de Contenido y Lenguas Extranjeras); Enseñanza superior en Reino Unido; Perspectiva docente
\end{abstract}

\begin{abstract}
One of the main characteristics of British Higher Education degrees where Spanish is taught is the distinction between language subjects where students learnt how to speak the language- and content subjects -where the history, literature, politics, etc. of the Spanishspeaking countries is discussed-. This article is based on the thematic analysis of interviews with university teachers of Spanish in the United Kingdom to explore whether the use of CLIL (Integrated Learning of
\end{abstract}


Content and Foreign Languages) can facilitate collaboration between teachers of both types of subjects.

Keywords: Spanish Language Teaching; Content and Language Integrated Learning (CLIL); Higher Education in the UK; teachers' views 


\section{Introducción}

La enseñanza de lenguas adicionales en Reino Unido da comienzo en la educación primaria y se extiende, si el aprendiz lo desea, hasta los niveles de especialización universitaria. El español, en concreto, se considera una lengua con una importancia y una utilidad internacionales (Palacios et al., 2019) y forma parte, por tanto, de las que cuentan con un mayor número de estudiantes entre aquellas que en inglés se conocen como MFL (Modern Foreign Languages, "Lenguas Modernas"; cf. Dobson 2020; Ministerio de Educación y Formación Profesional, 2020).

Con respecto a la educación superior, el español es, en la actualidad, la lengua más demandada entre los universitarios británicos para complementar sus estudios de grado (Ministerio de Educación y Formación Profesional, 2020: 600). Existen dos tipos principales de alumnos que llegan a la universidad británica para estudiar español: un perfil de estudiante responde al que ha continuado con su educación optativa en una lengua adicional y que posee lo que se conoce como un A-Level o Advance Level Qualification en lengua española, es decir, un diploma equivalente al Bachillerato español y que correspondería a un nivel B2, según el Marco Común Europeo de Referencia para las Lenguas (MCER; cf. Consejo de Europa, 2021[2001]); el segundo perfil de estudiante se correspondería con un alumno que, sin haber estudiado una lengua adicional desde los 14 años, vuelve a interesarse por el aprendizaje de una o varias lenguas adicionales durante su especialización universitaria (UCML, 2014).

Una vez que el alumno llega a la universidad, tiene que decidir qué tipo de estudios va a realizar: un Single Honours, es decir, una carrera que ofrece una especialización en una única área de estudio, por ejemplo, Hispanic Studies (el equivalente a la Filología Hispánica), un Combined Honours, esto es, un grado con doble titulación en los que se estudia, equitativamente, dos áreas diferentes, por ejemplo, Historia y Español, Económicas y Español, Relaciones Internacionales y Español, etc., o un Major/Minor, es decir, un grado en el que, igualmente, se ofrece una doble titulación, pero no de manera equitativa, sino que una de las áreas de estudio constituye el $75 \%$ de los créditos cursados y la otra, un $25 \%$. Por lo tanto, son diferentes las vías por las que un estudiante universitario accede a una carrera en la que puede estudiar tan solo español o español combinado con otras disciplinas.

Conviene aclarar, asimismo, cómo está configurada la enseñanza del español a nivel disciplinario. Según Pountain $(2019,247)$, la enseñanza de una lengua adicional en la universidad estaría conformada por tres componentes diferentes, pero interrelacionados, a los que denomina las tres eles: Lengua, Lingüística y Literatura. Dentro del currículum, estas tres eles quedan divididas en dos partes: el aprendizaje de la lengua adicional (primera ele) y lo que se suele llamar "contenido" (segunda y tercera eles). Debe precisarse además que dentro de "contenido" no solo se cubren áreas tradicionalmente relacionadas con la Filología, sino que también se incluye 
la docencia en otras áreas relacionadas con la dimensión cultural de la lengua como, por ejemplo, la Historia, la Política o el Arte (cf. Álvarez, Montoro, Campbell y Polisca, 2018: 8). Esta distinción resulta fundamental para comprender cómo está organizada la docencia universitaria del español en Reino Unido, ya que, por ejemplo, el aprendizaje de la lengua española y el aprendizaje de sus culturas se marca por la lengua de instrucción: en la mayoría de los centros universitarios en Reino Unido, las asignaturas de contenido son impartidas en la lengua del estudiante (en este caso, el inglés) y no en español (Pountain, 2019).

A esta distinción cabe añadir también la separación existente, en muchas universidades británicas, entre los docentes que imparten las asignaturas de español, que en numerosas ocasiones pertenecen al centro de idiomas de la universidad, y quienes imparten las asignaturas de contenido, que forman parte del departamento de Hispánicas de la Universidad (Pountain, 2019). Estas diferencias tienen también implicaciones importantes en términos de estabilidad laboral, remuneración económica y desarrollo profesional: en muchas ocasiones, mientras que los profesores adscritos a un departamento disfrutan de contratos indefinidos y oportunidades para desarrollar sus investigaciones, los profesores vinculados al centro de idiomas frecuentemente tienen contratos a tiempo parcial (y deben trabajar en más de un lugar a la vez) y la investigación no forma parte de sus obligaciones contractuales (cf. Álvarez, Montoro, Campbell y Polisca 2018, donde se analizan con detalle estas diferencias). Además, el que la enseñanza del español esté desligada de un departamento universitario explica en parte por qué las asignaturas de contenido no se imparten en la lengua meta.

Esta distinción tan tajante entre asignaturas de lengua y asignaturas de contenido presente en la enseñanza del español en las universidades británicas resulta llamativa en un momento en el parece que la enseñanza de lenguas adicionales aboga por enfoques abarcadores e integradores en los que la cultura $y$, sobre todo, por el desarrollo de las competencias comunicativas interculturales, que poseen una gran importancia dentro del aprendizaje de la lengua (Consejo de Europa, 2021[2001]). Por este motivo, a partir de la idea de que planear y plantear la enseñanza del español y sus culturas de manera integrada podría traer consigo mejoras respecto a la metodología que se utiliza en la actualidad, este trabajo se propone evaluar hasta qué punto la aplicación del enfoque AICLE (Aprendizaje integrado de Contenido y Lenguas Extranjeras) se halla implantado o podría implantarse en las carreras de lenguas en Reino Unido para contribuir a promover una enseñanza más integrada. En concreto, para los propósitos de este artículo, se explora la visión de un grupo de profesores de ELE con gran experiencia en la educación superior en Reino Unido. En particular, se busca responder a las siguientes preguntas de investigación: 
- ¿ ¿Cómo valoran los profesores de lengua española en universidades británicas la división entre las llamadas asignaturas de lengua y asignaturas de contenido?

- ¿ ¿Cuál es el grado de conocimiento de los profesores de ELE sobre el enfoque AICLE?

- ¿Cómo se posicionan los profesores universitarios de español en Reino Unido con respecto a la implantación del enfoque AICLE?

\section{Marco teórico}

\subsection{Origen, definición y características del enfoque AICLE}

El término CLIL (Content and Language Integrated Learning, por sus siglas en inglés) o AICLE (Aprendizaje Integrado de Contenidos y Lenguas Extranjeras) hace referencia al aprendizaje de una lengua adicional a través de la enseñanza de un contenido didáctico, de manera que la lengua adicional se convierte en una herramienta y no se ve solamente como una asignatura más dentro de un plan de estudios. Igualmente, cabe destacar que, entre lengua y contenido, no hay uno que tenga más peso que el otro, ya que se pretende conseguir un conocimiento amplio de los dos, por eso se habla de "aprendizaje integrado" (Eurydice, 2006).

El origen del enfoque AICLE se remonta a los años noventa y está muy ligado al Consejo de Europa. ${ }^{1} \mathrm{El}$ objetivo inicial de este enfoque era que, a partir del aprendizaje de la lengua y la cultura de otros países europeos, se redujera el efecto "barrera" que la lengua suponía entre ellos y, así, favorecer la unión pacífica que se planteó después de la Segunda Guerra Mundial. Este aprendizaje integrado resultó muy llamativo para ciertos países que, en aquel momento, buscaban el crecimiento de los planes de estudios a través de una lengua extranjera para la formación de ciudadanos europeos plurilingües. Como resultado, en la actualidad, el enfoque AICLE suele aplicarse en niveles de educación obligatoria, en asignaturas como matemáticas, artes plásticas, historia, música, ciencias o educación física, pero, en teoría, puede aplicarse a cualquier nivel educativo (Eurydice, 2006).

En particular, tal y como indica Do Coyle, (Working CLIL Digital, 2018), en muchos países de Europa pioneros en la propuesta de la aplicación del enfoque AICLE o de otros enfoques bilingües, la demanda de su implementación en el sistema educativo se hizo por parte de los profesores de lo que se denomina contenido, quienes buscaban motivar al alumnado con ello; por el contrario, en Reino Unido, se hizo por parte de los profesores de lengua, porque el aprendizaje de lenguas adicionales siempre ha supuesto un problema (Working CLIL Digital, 2018).

Al tratarse de una propuesta reciente, el enfoque AICLE se considera un movimiento dinámico, que se encuentra en constante evolución, sobre

\footnotetext{
${ }^{1}$ En concreto, nos referimos a la Resolución del Consejo, de 31 de marzo de 1995, relativa a la mejora de la calidad y la diversificación del aprendizaje y de la enseñanza de las lenguas en los sistemas educativos de la Unión Europea, Diario Oficial C 207 de 12.08.1995.
} 
todo a lo largo de los últimos años, por lo que carecemos de una definición cerrada de qué es; además, tampoco se ciñe a una metodología concreta. No obstante, podemos identificar una serie de estrategias pedagógicas que pueden servir para caracterizarlo (Montalto et al., 2016; Working CLIL Digital, 2018):

- El uso de la lengua adicional tiene un fin comunicativo y se presta más atención a que el alumno sepa comunicarse que la forma en la que lo hace. Por esa misma razón, la traducción de una lengua a otra no se considera AICLE, ya que este enfoque busca que el alumno sepa expresarse con autonomía y soltura en la lengua adicional y no dependa de la traducción desde su lengua materna para ello.

- Es aplicado dentro de metodologías activas y con ayuda de las TIC, ya que fomenta el aprendizaje colaborativo y cooperativo.

- Las dificultades de los alumnos son siempre tenidas en cuenta, ya que este enfoque afecta tanto a su rendimiento como a su autonomía. Además, la figura del profesor sirve como soporte al proceso de aprendizaje que estos llevan a cabo.

- La utilización de la lengua materna no está prohibida: se potencia la práctica de la lengua adicional, pero los alumnos pueden acudir a su lengua materna en caso de necesidad.

Además, el enfoque AICLE se nutre de los postulados de teóricos con gran influencia en los ámbitos de la educación y la enseñanza de lenguas, entre otras la Taxonomía de Bloom (revisada recientemente por Anderson y Krathwol, 2001) -que, aplicada a AICLE, se refiere a la realización escalonada de tareas (García 2013: 51)—, la metáfora del andamiaje (o scaffolding) vygotziana o la hipótesis del input de Krashen, (Baralo, 1999), que explicaría, en el enfoque AICLE, cómo puede plantearse la adquisición del nuevo input en la lengua adicional relacionado con las asignaturas de contenido.

En cuanto a las teorías más específicas en las que se fundamenta AICLE, en primer lugar, encontramos el Modelo de las 4 Ces (The $4 C^{\prime}$ 's Model, Coyle, 2005), según el cual existen cuatro dimensiones que conforman el enfoque AICLE que están presentes, en mayor o menor medida, en toda materia impartida en una lengua extranjera: contenido (aquello que se aprende), cognición (las habilidades mentales que se desarrollan), comunicación (el proceso de interacción) y cultura (comprensión de la identidad de las personas que hablan una lengua). En segundo lugar, cabe destacar el llamado tríptico del lenguaje (The Language Triptych: Coyle, Hood y Marsh, 2010), que habla del 1) lenguaje de aprendizaje (language of learning), que se relaciona con todo el lenguaje específico, la jerga y los términos clave correspondientes a las asignaturas estudiadas en la lengua adicional; 2) lenguaje para el aprendizaje (language for learning), aquel que el estudiante necesita para realizar las diferentes actividades que se desarrollan en el aula y a través del aprendizaje; 3 ) lenguaje a través del 
aprendizaje (language trough learning), el que el estudiante adquiere a partir de su exposición a un determinado input. Así, por ejemplo, en una clase de biología impartida en español a anglófonos, vocablos como mitocondria, celulosa o fotosíntesis formarían parte del lenguaje de aprendizaje, exponentes de argumentación o planteamiento de hipótesis corresponderían al lenguaje para el aprendizaje y que el alumno retenga una expresión como "Mañana más y mejor" por haberla escuchado en clase pertenecería al lenguaje a través del aprendizaje.

\subsection{Ventajas y limitaciones del enfoque AICLE}

Una de las mayores ventajas de la aplicación de AICLE es, naturalmente, el desarrollo de la interculturalidad. La visión de la identidad de los otros a través de la lengua es una de las capacidades que más se potencian y, además, la lengua extranjera proporciona a los estudiantes otras formas de expresión que no existen en su lengua materna y se favorece su competencia plurilingüe. Otra ventaja de este enfoque sería el aumento de la motivación de los alumnos. El uso de la lengua extranjera en otras materias hace que la visión de esta cambie, que les resulte más relevante y significativa y aumente su interés en la misma. Las clases de contenido en lengua extranjera proporcionan una visión auténtica de los idiomas y los alumnos pueden ver su uso más allá del hecho de tener una conversación banal o relacionada con su mundo cotidiano. La última ventaja sería la potenciación de la autonomía del alumno. A través del apoyo o andamiaje por parte del profesor, los alumnos son capaces de volverse independientes, lo cual puede desembocar en el aumento de su motivación, igualmente. Así, estas tres ventajas recuerdan a los criterios básicos que todo material didáctico debe cumplir: la autenticidad, la autonomía y la concienciación cultural. También cabe destacar que los alumnos de AICLE mejoran el conocimiento de la lengua adicional y las habilidades de comunicación que poseen de ella, haciendo que estos aprendan del mismo modo que lo haría un hablante competente y preparándolos para posibles futuros estudios y la vida laboral (Frydrychova, 2012).

Por otra parte, en cuanto a las limitaciones, cabe destacar el reto que supone su implementación en ciertos niveles de enseñanza: aunque AICLE es aplicable a cualquier nivel, hoy en día hay pocos ejemplos de aplicación en educación infantil y superior (Eurydice, 2006). Igualmente, otro elemento que puede limitar la aplicación de este enfoque es el profesorado y las necesidades del centro donde se establezca, puesto que la implantación de AICLE es más compleja que la enseñanza tradicional de lenguas (Eurydice, 2006).

\subsection{AICLE y la enseñanza del español}

Si bien AICLE es un enfoque que está en desarrollo desde los años noventa, su crecimiento no ha sido parejo en todas las lenguas. Mientras que el 
aprendizaje del inglés como lengua adicional ocupa el primer puesto en los estudios y la implantación de AICLE (Dobson, 2020; cf. asimismo Lorenzo, Trujillo y Vez, 2011 y, para el caso de España, Lasagabaster y Ruíz Zarobe, 2010 y Julián, 2013), apenas se encuentran ejemplos de materiales, propuestas educativas o centros de enseñanza del español como lengua extranjera (ELE) en los que se utilice. Esto se ve reflejado, por ejemplo, en el repositorio de actividades AICLE de Pérez (1997-2014), que apenas recoge materiales para otras lenguas que no sean el inglés.

No obstante, sí se han localizado algunas propuestas para la enseñanza y el aprendizaje del español en las que se intenta incorporar el enfoque AICLE, como, por ejemplo, la de Castro (2013), quien propone la integración de las pautas de una WebQuest a la metodología AICLE en la clase de ELE, o la de Lorente y Tirado (2019), quienes sugieren el empleo de lo que llaman un "AICLE moderado" $y$, a partir de una obra de teatro, desarrollan un curso de español. Además, entre las propuestas más recientes, cabe destacar la de García del Río y San Isidro (2016) para el tratamiento de contenidos relativos a las Ciencias Sociales en las escuelas de secundaria.

En cuanto al contexto de Reino Unido en concreto, según el Ministerio de Educación y Formación Profesional (2020), AICLE tiene una escasa representación y, pese a que se han llevado a cabo algunos proyectos AICLE a pequeña escala, la falta de recursos y ayudas por parte del gobierno han hecho que no se puedan sostener (Dobson, 2020: 515). Además, cabe destacar que los proyectos existentes, normalmente, están asociados a la educación primaria y secundaria (por ejemplo, las aulas ALCE, cf. Ministerio de Educación y Formación Profesional, 2020), lo cual coincide igualmente con el contenido de los programas y cursos de formación de profesores de ELE en metodología AICLE impartidos por entidades como el Instituto Cervantes o la Universidad Nebrija, que presentan estrategias y recursos aplicables a asignaturas como Ciencias Sociales, Ciencias Naturales, Tecnología o Matemáticas, propias de estos niveles educativos. Por lo tanto, puede concluirse que la formación universitaria británica carece de representación en estos proyectos, con la excepción de la Universidad de Aston (Birmingham), que sí cuenta con proyectos basados en este enfoque (Dobson, 2020).

\section{Metodología de la investigación}

\subsection{Enfoque de estudio}

Debido a la naturaleza social de este trabajo, se ha optado por la elección de una metodología de investigación cualitativa, para tratar de "entender, describir y algunas veces explicar fenómenos sociales 'desde el interior' de varias maneras diferentes" (Flick, 2007: 12). Además, de acuerdo con las características de las investigaciones cualitativas resaltadas por Taylor y Bogdan (1987), este estudio busca comprender los puntos de vista de los 
participantes en la investigación, por lo que no se toma ninguno como correcto o incorrecto y su objetivo no es que sea replicado, sino que sea válido desde el punto de vista empírico. A partir de estas premisas, se utiliza el estudio de caso como instrumento de investigación, ya que ofrece la posibilidad de hacer un examen detallado y diagnóstico de uno o varios fenómenos para comprender sus particularidades a través de los testimonios de los participantes en la investigación (Dorio, Sabariego y Massot, 2004) y se utilizan las fases del estudio de casos que proponen Montero y León (2002). Una vez recogida toda la información a través de las entrevistas y realizadas las transcripciones, para llevar a cabo el análisis de los datos, se optó por utilizar el análisis temático como técnica de investigación, por su adecuación a las investigaciones sociales de tipo cualitativo y por su flexibilidad para describir y organizar un tema. Además, permite la identificación de patrones comunes en los testimonios de los entrevistados para poder interpretarlos de manera conjunta (Braun y Clarke, 2006; Escudero, 2020).

\subsection{Instrumentos y recogida de datos}

Dado que el objetivo principal de la investigación es el análisis cualitativo de la opinión de los profesores universitarios de ELE en Reino Unido, se utilizó la entrevista semiestructurada como el instrumento de recogida de datos, pues permite que, a partir del guion preparado por el entrevistador (Tabla 1), los entrevistados pueden matizar y profundizar sus respuestas libremente, lo cual permite obtener información rica en matices (Dorio, Sabariego y Massot, 2004: 337).

\section{Tabla 1. Guion de la entrevista semiestructurada}

1. Hábleme de su experiencia laboral como profesor de ELE y cuánta de esa experiencia ha tenido lugar en Reino Unido como profesor universitario.

2. Desde su punto de vista, ¿̇cree que hay algún problema en la división que se produce entre lengua y contenido en las carreras de lenguas que se pueden estudiar en Reino Unido?

3. ¿Esta visión que posee ahora mismo del problema coincide con sus experiencias pasadas como enseñante? Es decir, ¿ha sido un problema del que se ha dado cuenta en seguida o lo ha ido viendo a medida que se desarrollaba su experiencia como docente universitario?

4. ¿Conoce el enfoque AICLE? [Si dice que sí, preguntarle ventajas y desventajas].

5. ¿Lo utiliza en sus clases? ¿Por qué sí/no?

6. [Si la respuesta es afirmativa]: ¿Cómo lo utiliza? ¿Podría darme un ejemplo concreto de aplicación?

7. [Si no lo utiliza]: ¿Consideraría utilizarlo en el futuro?

8. ¿Cree que la implementación de este tipo de enfoques puede ser parte de la solución al problema al que se enfrenta la enseñanza de lenguas en Reino Unido? Y si no es así, ¿dónde cree que está la solución?

9. [Si la respuesta anterior ha sido positiva] Por lo tanto, ¿cuál cree que debería ser el siguiente paso a seguir?: la implementación directa del modelo, es decir, pasar a dar directamente las clases de contenido en la lengua meta o, por el contrario, en lugar de hacer esa diferenciación tan marcada entre clases de lengua y clases de contenido, 
hacer que las clases de lengua también ofrezcan contenido igualmente para el beneficio del alumno?

10. ¿Cree que la impartición de contenido en la lengua meta, en este caso el español, puede suponer una pérdida de motivación de los alumnos?

\subsection{Participantes en la investigación}

A través de un muestreo no probabilístico por conveniencia, se contactó con profesores universitarios de ELE en Reino Unido. El principal requisito de selección de los participantes fue que contaran con una experiencia superior a diez años en docencia en educación superior en Reino Unido, ya que, por los objetivos del estudio, se buscaba que los participantes pudieran aportar una visión profunda, forjada desde la experiencia, sobre la implantación del enfoque AICLE en el contexto de análisis. Cinco de los docentes contactados accedieron a ser entrevistados.

Con respecto a las características demográficas de los informantes, tres se identifican como mujeres y dos como hombres. Ejercen como profesores de español en cuatro universidades británicas distribuidas por toda su geografía. Todos ellos son docentes experimentados, tal y como se requería, con una experiencia profesional universitaria de entre dieciocho y veintisiete años. Las entrevistas se realizaron de manera individual a través de la plataforma Zoom y fueron grabadas y transcritas para facilitar su análisis. Como primera fase del tratamiento de los datos, se asignó a cada docente un código para conservar su anonimato y solo una de las dos investigadoras conoce la identidad de los docentes entrevistados.

\subsection{Consideraciones éticas}

Todos los participantes recibieron una hoja informativa y dieron su consentimiento por escrito para participar en el proyecto. Las grabaciones de las entrevistas se encuentran en una carpeta en el ordenador de una de las investigadoras, protegido con contraseña, y se destruirán en diciembre de 2021. En las transcripciones, se ha eliminado toda la información que permite la identificación de los participantes, con el objetivo de poder compartirlas. Así, la primera pregunta del guion de la Tabla 1 no se incluye en las transcripciones de algunas entrevistas para salvaguardar el anonimato de los participantes, ya que algunos de ellos aportan información específica que puede dar lugar a su identificación. Dicha pregunta se ha utilizado para la comprobación de que todos los participantes de estudio contaban con el perfil adecuado para ejercer como tales. Solo aparece la transcripción de esta pregunta en aquellas entrevistas en las que se ha dado algún tipo de información relevante, no relacionada con la demografía del entrevistado. 


\section{Análisis y discusión de los datos}

\subsection{División entre lengua y contenido}

En cuanto a la primera pregunta de investigación, relativa a cómo valoran los docentes universitarios de español la división tan estricta entre asignaturas de lengua y asignaturas de contenido en el ámbito universitario británico, los participantes la atribuyen a factores contextuales, por ejemplo, a la manera en la que se conciben las lenguas como disciplina académica en Reino Unido. Así, se menciona cómo ya muchos colegios desaniman a los estudiantes a que elijan lenguas entre las materias de las que se examinan en las pruebas de acceso a la universidad ( $A$-Levels) por considerarse materias difíciles en las que supone un gran esfuerzo obtener una buena nota, en comparación con otras asignaturas (P4). Además, los entrevistados hablan de cómo la lengua se percibe como una herramienta o instrumento para acceder a una cultura, más que como una disciplina en sí misma: "el contenido es, prácticamente, la prioridad y la lengua es una destreza" (P2). Algunos entrevistados hablan incluso de materia de rango "inferior" o de "segunda categoría". El empleo de esta terminología para referirse a su propia disciplina resulta revelador con respecto a cómo los docentes entienden que se percibe su trabajo por la comunidad académica.

De hecho, los participantes ponen en relación esta concepción con el perfil profesional de los especialistas en lengua y quienes imparten asignaturas de contenido: queda patente en las entrevistas que esta división tan estricta entre lengua y contenido posee un impacto negativo en el estatus de los profesores de español que, sin embargo, no parece afectar a sus compañeros que enseñan materias de contenido. En particular, según los entrevistados, estas diferencias afectan a cuestiones como 1) el tipo de contrato y la remuneración económica percibida: “(...) también los contratos de los profesores de lengua suelen ser, en muchos casos de 10 meses, desde septiembre a junio. A pesar de ser fijos, son fijos discontinuos" (P4); 2) el estatus social, que uno de los entrevistados reporta en términos irónicos: “(...) es como que las clases de lengua las dan unos pobres seres y los catedráticos son los que saben mucho y dan las de contenido" (P3); 3) las diferencias en el conocimiento de la lengua entre los docentes de ambos perfiles: "no es nada raro conocer a investigadores muy top que tienen, lo que se llamaba antes las destrezas pasivas, que leen y entienden perfectamente, pero que luego comunicarse les cuesta un poco de trabajo" (P1). Estas ideas refrendan, entonces, las apuntadas por Álvarez, Montoro y Polisca, (2018) y Pountain (2019) con respecto a los perfiles docentes activos en las carreras de lenguas en Reino Unido. Al lado de estas diferencias, se apuntan también otras como la imposibilidad de acceder a la investigación o el surgimiento de conflictos relacionados con la organización interna del departamento (P2, P3, P4 y P5).

Con respecto al impacto de esta división en los estudiantes, en primer lugar, se cita un problema de gestión de expectativas: "muchos de 
ellos vienen a aprender español y una vez que llegan es cuando ya se dan cuenta de que también hay asignaturas de cine, también hay de literatura, también hay de género, etc.; pero eso les llega después. En realidad, ellos vienen con la idea de aprender español y se encuentran con que tienen 20 créditos de los 120 de cada año" (P1). Esto genera en ocasiones un descontento entre los estudiantes que tiene impacto sobre todo en las clases de lengua, puesto que juzgan que no están aprendiendo lo suficientemente rápido. En segundo lugar, el alumnado se encuentra con una segmentación en su especialización provocada por la diferente categorización de las asignaturas que componen su plan de estudios y por la formación en dos idiomas diferentes (español e inglés), que resulta en ocasiones en la incapacidad de transferir las competencias adquiridas de unas asignaturas a otras: “(...) Por ejemplo, nos encontramos con problemas a veces donde, a mí me comentan compañeros: 'Si es que llega el momento de participar en los seminarios y, como queremos que sea en español, nadie habla. Y es que no tienen el nivel'. Y digo: 'Bueno, pero ¿cómo puede ser que en la clase de lengua hablen sin ningún problema?'” (P2). Es interesante además destacar que varios entrevistados se refieren a su propia experiencia como alumnos de Filología Inglesa para indicar que, a lo largo de sus estudios, todas las asignaturas se impartían en inglés (P1, P3, P5), lo que puede tomarse como un indicativo de su creencia en la posibilidad de impartir clases de contenido en la lengua meta de manera exitosa.

Por tanto, todos los entrevistados valoran de manera negativa la división tan estricta existente entre lengua y contenido y destacan que no solo plantea problemas pedagógicos que impactan en la adquisición de competencias por el alumno, sino que también tiene implicaciones para los profesores de español que afectan al desempeño de su trabajo, ya que sus declaraciones muestran, en general, un sentimiento de falta de reconocimiento de su labor y su disciplina, si bien algunos de ellos se declaran optimistas y afirman, por ejemplo, que en ocasiones puede accederse a la carrera investigadora con un perfil de profesor de lengua (P4). El punto de vista expresado por los docentes con respecto a esta cuestión sirve para enmarcar su visión del enfoque AICLE y su opinión acerca de su posible implantación en las universidades británicas.

\subsection{Conocimientos sobre el enfoque AICLE}

En cuanto a la percepción del enfoque AICLE por parte de los entrevistados, cabe destacar que todos ellos se revelan interesados en su aplicación y parecen conocer en qué consiste y cómo puede implantarse. Respecto a la definición del enfoque AICLE, si bien todos los participantes en el estudio resaltan estar familiarizados con él, se perciben muchos matices cuando se refieren al enfoque AICLE, de manera que no se aporta una única definición de este. Según los testimonios de los entrevistados, esta metodología se caracteriza por el uso del español con el objetivo de aprender un contenido y que, a través de este, se consiga un amplio dominio de la lengua meta ("Es 
muy importante practicar la lengua a través de los distintos aspectos del currículum y hacer que la lengua sea lengua vehicular de la transmisión del contenido", P4) y la necesidad de un andamiaje para poder implantarlo, ya que AICLE no implica solo dar clase de contenido en la lengua meta, sino la adquisición de determinadas destrezas por parte del alumnado, a quien el profesor ha de guiar para que dicha adquisición se produzca con éxito: “(...) Para mí, CLIL, en ningún momento, es dar la clase en español. Requiere un andamiaje, requiere un trabajazo... (...) $Y$ es que esos puentes y esa transferencia de destrezas, si tú no las incorporas y no las prácticas y ellos no las adquieren, no surgen de manera espontánea". (P2) También se alude a su relación con la enseñanza y el aprendizaje basados en contenidos (P1).

De manera implícita, los entrevistados también muestran conocer las teorías más específicas en las que se asienta AICLE, puesto que, por ejemplo, uno de los participantes afirma que explica a sus alumnos en qué consiste esta metodología para favorecer su implicación en las clases y declara que "le explicas en qué consiste, le explicas las 4 Ces de Coyle, por ejemplo, le explicas las diferentes habilidades para que sepan un poco qué es lo que se espera de ellos y para qué estamos haciendo esto". (P5). Con respecto a otras teorías de las que también se nutre el enfoque AICLE, como, por ejemplo, la del procesamiento del input de Krashen o la Taxonomía de Bloom, es destacable, que, aunque a través de los testimonios de los entrevistados se puedan llegar a distinguir observaciones relacionadas con ellas (P2 y P5), estas no se mencionen explícitamente entre aquellas que consideran importantes para la implantación de AICLE en el aula de ELE.

El interés y la experiencia de los entrevistados en la metodología AICLE posee, de acuerdo con sus testimonios, varios orígenes: algunos de ellos mencionan sus propias experiencias como estudiantes como modelo apropiado que puede seguirse (P1, P2, P3); también indican que la formación en este enfoque procede de lecturas - aunque no se cita ninguna en concreto- (P2) y se refieren algunas experiencias previas de aplicación de AICLE de las que conocen sus resultados: "entiendo por qué hay tantos ejemplos de modelos que han fallado en este enfoque; desde los primeros experimentos en Canadá, los cursos bilingües de los que habla Mary Swain y todos estos, hasta lo más reciente que pasa ahora en España donde profesores y padres se quejan de que los niños no aprenden ni una cosa ni la otra" ( $P 1)$. Además, se alude a la preeminencia del inglés como lengua extranjera en la implantación de este enfoque (P1).

No obstante, de las palabras de los entrevistados se deduce que su principal fuente de conocimiento de este enfoque procede de su experiencia directa con AICLE: de hecho, todos los participantes en el estudio mencionan que lo utilizan en mayor o menor medida. Algunos de ellos hablan de "píldoras CLIL" en sus propias asignaturas, mediante el diseño de sus propios materiales a partir de este enfoque (P1, P2, P4, P5) o como refuerzo a las asignaturas de contenido (P1, P2, P3, P5). En este último caso, se citan varias iniciativas, entre ellas talleres en los que se trabajan sobre todo destrezas 
capacitadoras para que los alumnos puedan comprender las fuentes y textos para el input que se utilizan en las asignaturas:

Creé una serie de talleres de nivel A1 [...] La idea de que el alumno tome confianza a la hora de enfrentarse un texto en español, aunque no tenga ese nivel. Desde el uso del diccionario a escribir, bueno, pues si hasta ahora tú solo has aprendido a escribir en presente y en pretérito perfecto, pues, bueno, ¿por qué no lo vas a hacer? Que ese vocabulario que en teoría es de un nivel A1, A2, B1, B2, no sea un impedimento para ti con el diccionario, pues aprendo a utilizar el diccionario y a escribir un texto, aunque sea en presente histórico; no nos hace falta más... enfrentarte a videos que en un principio no están adaptados y que, bueno, con subtítulos y con una tipología de actividades para explotar ese video que sea adecuada para ese nivel; pero nunca enseñando la gramática de manera explícita. Ahí no existe eso. Para eso está ya la clase de lengua. (P2)

Es interesante observar que los participantes mencionan también cierta apertura de todos los docentes a la colaboración respecto a épocas anteriores (P2, P3), si bien en ocasiones apuntan a motivos extraacadémicos como justificación a este interés por integrar lengua y contenido, entre otros favorecer la sostenibilidad de los grados en lenguas en Reino Unido (P2).

\subsection{Posible implantación del enfoque AICLE}

En cuanto al posicionamiento de los entrevistados con respecto a la implantación del enfoque AICLE, se observa que todos ellos tienen experiencia directa en su empleo y se muestran partidarios de su aplicación en las aulas universitarias británicas. De hecho, mencionan más ventajas que inconvenientes en su posible aplicación (un participante indica: "¿desventajas? Ninguna”, P3); es más, uno de los participantes considera que "en nuestro contexto de enseñanza universitaria, creo que debería ser razonablemente fácil implementarlo" (P1). También se comenta la posibilidad de implantarlo de manera progresiva, mediante distintos tipos de tareas (como portafolios o actividades de mediación) hasta el empleo exclusivo de la metodología AICLE en los niveles avanzados correspondientes al último año de carrera $(\mathrm{P} 1, \mathrm{P} 2)$. Un aspecto que parece importante para los docentes es que el empleo del enfoque AICLE se alinee con las tareas de evaluación, para evitar posibles penalizaciones por el nivel de lengua de los estudiantes en las asignaturas de contenido: "se trataría de ponerse de acuerdo en el tipo de criterios de evaluación o incluso de rúbricas que se utilicen [...] en las clases de contenido se podría [...] valorar que, si alguien tiene la capacidad de utilizar la lengua meta, que la utilice, y que se le dé parte de la nota con la lengua meta y parte de la nota por el contenido" (P1). También se indica que es necesario que las instituciones se impliquen y que exista una estrategia y una estructura de implantación del enfoque AICLE, puesto que parece demostrado que iniciativas individuales de profesores no suelen dar resultados (P2, P5). 
Entre los principales argumentos que se esgrimen para mostrarse a favor de la implantación de la metodología AICLE, se valora el desarrollo de competencias transversales y la autonomía del alumno (P5), así como el potencial de aumentar la motivación de los estudiantes por el aprendizaje de la lengua (P1, P2, P3, P5):

Les encanta el poder estar escribiendo en español de cuestiones de contenido de las que normalmente no tienen la oportunidad de establecer esa relación; y el verse hablando de Derecho, de especialmente de esas partes con la que se sienten tan orgullosos porque ha sacado muy buena nota... [...] ese puente no va solamente de la Literatura Española a la clase de Lengua Española, sino de la clase de Derecho Internacional a la de Lengua Española. Luego, yo creo que es súper motivador para el estudiante y a mí me encanta verlos. Quieren hablar de lo que ellos están haciendo y poderlo contar. (P2)

No obstante, varios de los entrevistados se refieren también a los posibles retos que plantearía el empleo de esta metodología en las universidades británicas. En concreto, señalan, en primer lugar, la falta de formación del profesorado (P1, P3), lo que coincide con las conclusiones del informe Eurydice (2006), donde se afirma que el nivel de formación de los profesores puede limitar la implantación del enfoque o puede dar lugar a una situación en la que docentes poco experimentados en uno de los dos ámbitos comiencen a impartir clase de un contenido o en una lengua que no dominan. También se alude a posibles desafíos institucionales, como permitir que los docentes con cargas lectivas ya de por sí elevadas inviertan tiempo en el diseño de materiales basados en la metodología AICLE (P5) o el asumir ciertos riesgos económicos:

(...) Claro, ahí también hay una razón económica, porque ese módulo... se ofrecen muchos módulos, no hay muchos profesores y tú quieres que tu módulo se abra y que haya suficientes alumnos. Entonces, ¿qué haces? Si tú lo ofreces en inglés y es un módulo sobre, no sé, sobre la esclavitud en el mundo hispanohablante o la esclavitud en el Caribe; quieres que te venga estudiante de Historia o de Relaciones Internacionales también a hacértelo. Si es 1 o 2, pues 1 o 2 más. Estamos hablando aquí de economía, de dinero, no solo estamos hablando de enseñanza, ¿de acuerdo? Entonces, ese es el problema. Por eso se dan muchos módulos en inglés, para no cerrárselo al resto de los estudiantes de carreras afines. (P5)

Asimismo, se mencionan posibles dificultades para los estudiantes. En primer lugar, el que en las aulas haya alumnos con perfiles muy heterogéneos que avanzan en su aprendizaje del español a ritmos distintos puede suponer un problema para el empleo de AICLE (P1, P2). Además, aquellos estudiantes $a b$ initio, que ingresan en un grado universitario sin conocimientos previos de español, pueden sentirse desmotivados si desde el principio toda la materia se imparte en la lengua meta (P1, P3), si bien 
otros entrevistados afirman que es aplicable a cualquier nivel de competencia mediante al andamiaje y la adaptación que conlleva (P2, P4 y P5). Además, uno de los entrevistados indica que, para paliar esta posible carencia, los textos pueden ofrecerse en ambas lenguas y que el estudiante elija cómo prefiere leerlos (P1); también se alude a la función del profesor, quien puede reforzar, a través de tutorías, a aquellos alumnos que lo necesiten (P5). Otras posibles dificultades asociadas a los estudiantes son que quizá el empleo de este enfoque aumenta en principio la carga de trabajo por asignatura (P5), que en ocasiones resulta complicado articular un pensamiento complejo en la lengua meta, lo que puede traer problemas en las asignaturas de contenido (P1) y que, con relación a esto, se desconoce si la aplicación de la metodología AICLE puede suponer un detrimento en la profundidad con la que se tratan los temas de contenido (P1, P2).

Por lo tanto, la postura de los participantes en la investigación con respecto al enfoque AICLE es muy alentadora y todos ellos están a favor de su implementación en estudios universitarios, aunque no haya un consenso general sobre cómo llevarlo a cabo y se reconozcan distintos obstáculos institucionales y pedagógicos que deben estudiarse antes de proponer un modelo de implementación. Por ejemplo, surgen los interrogantes de si debiera aplicarse el enfoque AICLE en todos los cursos, con independencia del nivel de competencia de los estudiantes, y si resulta posible utilizar un mismo modelo en todos los centros de educación superior en Reino Unido.

\section{Conclusiones}

Este estudio se proponía trazar un estado de la cuestión sobre la implantación del enfoque AICLE en las universidades británicas a partir de las opiniones de un grupo de docente que enseña español en este contexto. Los resultados muestran que la división entre lengua y contenido que caracteriza de manera tradicional la enseñanza de lenguas en la educación superior en Reino Unido se muestra como una de las principales dificultades a las que se enfrentan los profesores de lengua, muchos de ellos con una categoría profesional, estatus social y carga docente muy diferentes de las de sus colegas que imparten asignaturas de contenido y esto supone un obstáculo en la posible implantación del enfoque AICLE. Los profesores demuestran que lo conocen, sobre todo mediante experiencias directas, y se manifiestan a favor de su implantación en los grados universitarios, siempre y cuando se tomen en consideración determinadas dificultades que afectan a los estudiantes, a los docentes y a las instituciones en las que se imparten estas materias. Es más, los docentes de ELE demuestran, mediante ejemplos concretos, que en las clases de lengua ya se integra el contenido, por lo que el escollo parece encontrarse en las asignaturas denominadas de contenido. Con relación al posible impacto negativo de AICLE en los estudiantes de la ruta ab initio y los alumnos procedentes de otras carreras universitarias que estudian español como minor, de manera que tendría que 
pilotarse la implantación de esta metodología en estos contextos y observar los resultados y las reacciones de estudiantes con este perfil.

Por lo tanto, entre las posibles soluciones para tratar de integrar la enseñanza del español y las asignaturas denominadas "de contenido" en las universidades británicas, cabría sugerir que las clases de contenido se impartan en español, con elementos tomados del enfoque AICLE. Para ello, sería necesaria una colaboración entre ambos grupos de docentes, ya fuera dentro de las propias clases de contenido o en talleres adicionales, lo cual haría que ambos grupos de materias estuvieran unidos y fueran totalmente complementarios. Además, debe notarse que en este estudio se recaban las opiniones de los profesores de lengua: para poder trazar un panorama completo ante una posible implantación del enfoque AICLE, convendría consultar a los profesores de las asignaturas de contenido y ver cuáles son las ventajas e inconvenientes de esta metodología desde su punto de vista también y en qué aspectos coinciden con las ideas de los docentes representados en este estudio.

\section{Bibliografía}

Álvarez, I., Montoro, C., CAMPbell, C. y PolisCA, E. (2018): Language Provision in UK MFL Departments 2018 Survey. University Council of Modern Languages: Londres.

BARALO, M. (1999): La adquisición del español como lengua extranjera. Arco/Libros: Madrid.

Braun, V. y Clarke, V. (2006): "Using thematic analysis in psychology". Qualitative Research in Psychology, 3 (2), pp. 77-101.

CONSEJO DE EUROPA (2021[2001]): Marco común europeo de referencia para las lenguas: aprendizaje, enseñanza, evaluación. Volumen complementario. Ministerio de Educación y Formación Profesional e Instituto Cervantes: Madrid.

COYLE, D (1999): "Supporting students in content and language integrated learning contexts: planning for effective classrooms". J. MASIH (ed.), Learning through a foreign language: models, methods and outcomes. Centre for Information on Language Teaching and Research: Londres, pp. 46-62.

COYLE, D. (2005): "Developing CLIL: Towards a Theory of Practice". Monograph 6, APAC: Barcelona.

CoYle, D., HOOD, P. Y MARSH, D. (2010): CLIL. Content and Language Integrated Learning. Cambridge University Press: Cambridge.

Custodio, M. y Caballero-García, P. A. (2016): “AiCLE: Enfoque alternativo para el desarrollo del vocabulario en el aula de español como lengua extranjera". MarcoELE: Revista de didáctica ELE, 23, disponible en https://marcoele.com/aicle-enfoque-alternativo-para-el-desarrollodel-vocabulario/

DOBSON, A. (2020): "Context is everything: reflections on CLIL in the UK". The Language Learning Journal, 48 (5), pp. 508-518. 
DoRIO, I.; SABARIEGO, M. y MASSOT, I. (2004): “Características generales de la investigación cualitativa". R. BISQUERRA (coord.), Metodología de la investigación educativa. La Muralla: Madrid.

ESCUDERO, C. (2020): "El análisis temático como herramienta de investigación en el área de la Comunicación Social". La Trama de la Comunicación, 24 (2), pp. 89-100.

EURYDICE, la Red Europea de Información sobre Educación (2006): "Aprendizaje Integrado de Contenidos y Lenguas (AICLE) en el contexto escolar europeo", Ministerio de Educación y Ciencia, Gobierno de España, Madrid, https://clil.files.wordpress.com/2009/04/clil-en-europa.pdf

FLICK, U. (2007): El Diseño de Investigación Cualitativa, ([trad.] T. del Amo y C. Blanco). Ediciones Morata: Madrid.

FRYDRYCHOVA, B. (2012): "CLIL and the teaching of foreign languages", Procedia - Social and Behavioral Sciences, 47, pp. 572-576.

García del Río, C. y SAN ISIDRO, F. X. (2016): ¿Cristóbal Colón usaba GPS? Tareas AICLE en Español. Ciencias Sociales. A2-B1. Difusión: Barcelona.

GARCíA, S. (2013): "Three frameworks for developing CLIL materials in infant and primary education". Encuentro: Revista de investigación $e$ innovación en la clase de idiomas, 22, pp. 49-53.

HARROP, E. (2012): "Content and Language Integrated Learning (CLIL): Limitations and possibilities". Encuentro: Revista de investigación e innovación en la clase de idiomas, 21, pp. 55-70.

JULIÁN, C. (2013): La coordinación de equipos docentes en centros bilingües de secundaria. Un estudio de casos. Tesis doctoral inédita, Universidad de Sevilla.

LASAgABASTER, D. y RuÍz ZAROBE, Y. (2010): CLIL in Spain: Implementation, Results and Teacher Training. Cambridge Scholars Publishing: Newcastle upon Tyne.

LeÓN, O., Montero, I. (2002): Métodos de Investigación en Psicología y Educación. McGrawHill: Madrid.

LORENTE, P. y TIRADO, A. M. (2019): "AICLE (CLIL) y teatro en una unidad didáctica de ELE. Max Aub: De algún tiempo a esta parte", Revista Mosaico, https://dial.uclouvain.be/pr/boreal/en/object/boreal\%3A209075/d atastreams

LORENZO, F., TRUIILLO, F. y VEZ, J.M. (2011): Educación bilingüe. Integración de contenidos y segundas lenguas. Editorial Síntesis: Madrid.

Martín PerIS, E., dir. (2005): Diccionario de términos clave de ELE. Instituto Cervantes: Madrid.

Ministerio de Educación y Formación Profesional (2020): El mundo estudia español. Secretaría General Técnica, Gobierno de España, disponible en https://sede.educacion.gob.es/publiventa/el-mundo-estudiaespanol-2020/ensenanza-lengua-espanola/24945 
Montalto, S., Walter, L., Theodorou, M. y Chrysanthou, K. (2016): Guía CliL, Clil4u,

https://www.languages.dk/archive/clil4u/book/CLIL\%20Book\%20ES .pdf

PÉREZ, I. (1997-2014): Repositorio de recursos CLIL [página web]. http://www.isabelperez.com/clil.htm

Pountain, C. (2019): "Modern Languages as an academic discipline: the linguistic component". Language, Culture and Curriculum, 32 (3), pp. 244-260.

TAYLOR, S. J. y BOGDAN, R. (1987): Introducción a los métodos cualitativos de investigación. Paidós: Barcelona.

UCML (2014): Language Teaching Survey Report, University Council for Modern Foreign Languages: Londres https://university-councilmodern-languages.org/wp-content/uploads/2021/05/UCMLlanguage-survey-report-2014.pdf

UNIVERSITY OF LEEDS (2020): "SPPO1045 The Historical Development of Spain and Hispanic America". En 2020/21 Undergraduate Module Catalogue, disponible en http://webprod3.leeds.ac.uk/catalogue/dynmodules.asp?Y=202021 \&M=SPPO-1045

Working CLIL DIGITAL (2018): "Professor Do Coyle - How do you define CLIL?", [Vídeo online]. https://www.youtube.com/watch?v=URNsMhexQpM\&t=169s 